Wilfrid Laurier University

Scholars Commons @ Laurier

Psychology Faculty Publications

Psychology

2010

\title{
The use of paraphrasing in investigative interviews
}

Angela Evans

Unversity of Toronto, aevans@brocku.ca

Kim P. Roberts

Wilfrid Laurier University, kroberts@wlu.ca

Heather L. Price

University of Regina

Candyce P. Stefek

Follow this and additional works at: https://scholars.wlu.ca/psyc_faculty

Part of the Psychiatry and Psychology Commons

\section{Recommended Citation}

Evans, A.D., Roberts, K.P., Price, H.L., \& Stefek, C. (2010). The use of paraphrasing in investigative interviews. Child Abuse \& Neglect, 34, 585-592. DOI: 10.1016/j.chiabu.2010.01.008

This Article is brought to you for free and open access by the Psychology at Scholars Commons @ Laurier. It has been accepted for inclusion in Psychology Faculty Publications by an authorized administrator of Scholars Commons@ @aurier. For more information, please contact scholarscommons@wlu.ca. 


\title{
Running Head: PARAPHRASING IN CHILD INTERVIEWS
}

The use of paraphrasing in investigative interviews

\author{
Angela D. Evans ${ }^{1}$ \\ Kim P. Roberts ${ }^{2}$ \\ Heather L. Price ${ }^{3}$ \\ Candyce P. Stefek ${ }^{2}$ \\ University of Toronto $^{1}$, Wilfrid Laurier University ${ }^{2}$ and University of Regina ${ }^{3}$
}

\footnotetext{
${ }^{2}$ Corresponding author: Kim P. Roberts, $\mathrm{PhD}$, Wilfrid Laurier University, Department of Psychology, 75 University Avenue West, Waterloo, ON, N2L 3C5, Canada; kroberts@wlu.ca;
}

Acknowledgements: A portion of the results were presented at the biannual meeting of the American Psychology and Law Society, in Jacksonville Florida, in May 2008. 


\begin{abstract}
Objective

Young children's descriptions of maltreatment are often sparse thus creating the need for techniques that elicit lengthier accounts. One technique that can be used by interviewers in an attempt to increase children's reports is 'paraphrasing', or repeating information children have disclosed. Although we currently have a general understanding of how paraphrasing may influence children's reports, we do not have a clear description of how paraphrasing is actually used in the field.

Method
\end{abstract}

The present study assessed the use of paraphrasing in 125 interviews of children aged 4 to 16 years conducted by police officers and social workers. All interviewer prompts were coded into four different categories of paraphrasing. All children's reports were coded for the number of details in response to each paraphrasing statement. Results

'Expansion paraphrasing' (e.g., "you said he hit you. Tell me more about when he hit you") was used significantly more often and elicited significantly more details, while 'yes/no paraphrasing' (e.g., “he hit you?”) resulted in shorter descriptions from children, compared to other paraphrasing styles. Further, interviewers more often distorted children's words when using yes/no paraphrasing, and children rarely corrected interviewers when they paraphrased inaccurately. Conclusions and Practical Implications 
Investigative interviewers in this sample frequently used paraphrasing with children of all ages and, though children's responses differed following the various styles of paraphrasing, the effects did not differ by the age of the child witness. The results suggest that paraphrasing affects the quality of statements by child witnesses. Implications for investigative interviewers will be discussed and recommendations offered for easy ways to use paraphrasing to increase the descriptiveness of children's reports of their experiences. 
The use of paraphrasing in investigative interviews

When interviewed in an open-ended manner, children can provide testimony that is equally accurate, or even superior to that of adults (Goodman \& Reed, 1986). However, young children's descriptions of maltreatment are often sparse, creating the need for techniques that elicit lengthier accounts from children (McCauley \& Fisher, 1995a; Saywitz \& Snyder, 1996). Effective techniques for eliciting accurate and detailed accounts from children have been studied for many years including the use of structured interviews, interview location, and the use of anatomically correct dolls (Edwards \& Forman, 1989; Gordon, Ornstein, Nida, Follmer, 1993; Samra, \& Yuille, 1996; Shrimpton, Oates, \& Hayes, 1998; Sternberg, Lamb, Esplin, Orbach, \& Hershkowitz, 2002). One specific technique that can be used by interviewers in an attempt to increase the length of children's reports is 'paraphrasing'. For the purposes of this paper, paraphrasing is defined as repeating information a child has disclosed in whole or in part. For example, if a child stated, "She hit me", an interviewer may restate the information as "She hit you." Previous research has experimentally assessed the influence of different types of paraphrasing on children's event reports and found that some styles of paraphrasing are indeed more beneficial than others (Evans \& Roberts, in press). However, little is known about the prevalence and potential effects of different types of paraphrasing in actual investigative interviews.

To date there has been one study that has explored the use of paraphrasing in investigative interviews of child witnesses. Roberts and Lamb (1999) assessed 'distortions' that naturally occurred in investigative interviews. Distortions were defined as any word, phrase or utterance by the interviewer that changed or contradicted what the 
child had said (e.g., if the child said "It happened by the cafeteria", one interviewer replied with, "It happened inside the cafeteria."). Only one third of such distortions were corrected by children and, when not corrected, interviewers continued to use the distorted details throughout the remainder of the interview. However, only a small sample of investigative interviews was used $(n=68)$ and the interviewers were not trained in openended interviewing. In addition, no prevalence data was reported on the number of paraphrases used, the style of paraphrasing used, or children's utterances in response to accurate paraphrases.

Although no studies to date have specifically investigated the use of different styles of paraphrasing in investigative interviews, one study has experimentally manipulated paraphrasing styles in a laboratory study. In Evans and Roberts (in press) children participated in a staged event and were interviewed about the event one week later by an interviewer who either paraphrased children's statements by incorporating them into open-ended prompts (expansion paraphrase condition: in response to a child saying "I dressed up" the interviewer would say, "You dressed up, tell me more."), paraphrased children's statements and used intonation to turn it into a yes/no question (yes/no paraphrase condition: "You dressed up?") or only used open-ended prompts (control condition: "Tell me more."). Results indicated that children in the expansionparaphrasing condition reported significantly more details overall and proportionally more accurate details than children in the yes/no-paraphrase condition. Thus, the style of paraphrasing used by interviewers influenced children's reports, at least in these laboratory interviews. 
While there has been little research completed on paraphrasing in investigative interviews, previous literature examining the influence of question format on children's responses can provide insight into the influence of paraphrasing on children's reports. In investigative interviews, cued invitations (i.e., 'Tell me more about [something the child has mentioned]') have been found to elicit more information than simple invitations alone (i.e., 'Tell me more') (Lamb et al., 2003; Orbach \& Lamb, 2000). Some researchers suggest that the increased information is a result of the cue (the specific detail the interviewer is referring to) focusing children's attention and fostering elaboration of essential or central details (Orbach \& Lamb, 2000). The efficacy of these recall cues are dependent on accurate paraphrasing (repeating) of children's utterances. In addition to recall cueing, another theoretical possibility is that the format of the cued invitation motivates children to report more information. By including the cue, children may feel as though the interviewer is listening to them and cares about what they are saying. In turn, children may feel motivated to provide additional information.

Evans and Roberts (in press) proposed two ways that paraphrasing may motivate children to expand their responses. First, paraphrasing may encourage rapport between the child witness and interviewer. When the interviewer restates what the child just said, it highlights to the child that the interviewer is listening to the child's disclosure and cares about what the child has to say. This may result in the child feeling supported and motivated to disclose additional information to an interviewer that they trust. Second, paraphrasing may transfer control to children. This idea of transferring the control to the child is a component of the Revised Cognitive Interview (Fisher \& Geiselman, 1992). Paraphrasing may be effective in transferring control because it highlights the child's 
statement over the interviewer's. Previous studies have found that children interviewed using the Revised Cognitive Interview protocol tend to report 46 to $84 \%$ more correct information than children interviewed with a standard interview technique (McCauley \& Fisher, 1995a, b).

However, we do not have a clear understanding of how paraphrasing is actually used in the field and what effects different styles of paraphrasing have on children's testimony. In the current study, we investigated the use of paraphrasing in 125 child maltreatment investigative interviews. Four styles of paraphrasing were assessed based on Evans and Roberts'scheme (in press; see Method).

The present study attempts to answer four primary questions. First, how often do investigative interviewers use each style of paraphrasing? We then examined which style of paraphrasing elicits the most information in investigative interviews. It is hypothesized that significantly more details will be reported in response to an expansion (open-ended) style paraphrase than yes/no paraphrasing (Evans \& Roberts, in press). As indicated by researchers such 'cued invitations' prompt children to provide additional information (e.g., Sternberg et al., 2001; Hershkowitz, 2001) and, as argued here, may motivate children because the interviewer is clearly attentive and interested (as revealed in the repetition of the child utterances). In contrast, yes/no paraphrasing simply requires a yes or no response and does little to maintain rapport and motivate children to expand on their responses. Rather, it can appear to be a test (Roberts et al., 2004).

Third, we assessed whether the effects of paraphrasing varied by age. Previous studies assessing the use of paraphrasing have not found significant age differences (Roberts \& Lamb, 1999; Evans \& Roberts, in press). Additionally, work conducted on 
the NICHD investigative interviewing protocol, which includes paraphrasing in the form of cued invitations, has been found to be effective in improving both younger and older children's reports. Further, there are few developmental differences in investigations of motivated reporting (e.g., Roebers \& Fernandez, 2002). Thus, paraphrasing was not expected to influence younger and older children's responses differently (e.g., Lamb et al., 2003). However, as different types of paraphrasing were investigated in this study (Roberts \& Lamb, 1999; Evans \& Roberts, in press), a wide age range (4-16 years) was included to detect any age differences. Finally, we examined how often investigative interviewers inaccurately paraphrased children's utterances and whether children corrected these inaccurate paraphrases. Based on previous findings, we hypothesized that children would rarely disagree with the inaccurate paraphrases (Roberts \& Lamb, 1999; Hunt \& Borgida, 2001).

Method

\section{Sample}

One hundred twenty-five transcripts from child abuse interviews conducted by 13 police officers and social workers over a 6-month period while participating in a training course on open-ended interviewing techniques were analyzed. The Institutional Review Board reviewed and approved the project and all interviewers and interviewees (or proxy) gave consent for their interviews to be included in this project. Twenty-three of the children were 4 to 6 years old $(M=5.35, S D=.83), 35$ were 7 to 9 years old $(M=8.06$, $S D=.77), 27$ were 10 to12 years old $(M=11.15, S D=.77), 30$ were 13 to 16 years old $(M=14.33, S D=1.03)$; the remaining 10 participants' ages were unknown. Of the 125 participants, $50.4 \%$ were male, $46.2 \%$ were female, and the remaining $3.2 \%$ were 
unrecorded. Approximately 44\% of allegations were of hitting, 14\% sexual assault, 17\% observing fighting, $9 \%$ general violence, and the remaining were other forms (e.g., yelling or fighting). Sixty percent of cases were allegations of repeated abuse. Additionally, the alleged perpetrator in the majority (67\%) of the cases was the child's parent, $10 \%$ were an adult acquaintance, $4.3 \%$ were a stranger, $4.3 \%$ involved multiple perpetrators, and the remaining were other relationships (e.g., teacher or sibling)

The interviews typically began by informing the children that it is okay to say, "I don't know", followed by a formal rapport-building phase. The interviewer then transitioned to the substantive phase where the child was encouraged to describe their experiences. Interviewers had received training in using open-ended interviewing and employed several different techniques (open-ended prompts, facilitators such as "uhhuh", as well as paraphrasing). However, no explicit instructions were given to interviewers on when or how often to paraphrase children's responses. Prior to receiving training, social workers' overall experience interviewing children ranged from 0.50 to 17 years $(M=4.33, S D=4.99)$. The participating police had been officers for 11 and 18 years and one had experience interviewing children for a year, while the other had three years experience interviewing children. The mean length of interviews was approximately 25 minutes ( $M=25$ minutes and 45 seconds; $S D=12.30$ minutes).

\section{Coding}

\section{Paraphrases}

Each transcript was coded for the use of paraphrases by the interviewer. Paraphrases were only coded if the interviewer's utterance ended in a paraphrase. Paraphrases were 
coded into four different styles including: yes/no paraphrase, expansion paraphrase, simple paraphrase or summary paraphrase ${ }^{1}$.

a) Yes/No paraphrasing occurred when the interviewer restated the child's utterance in the form of a question that could be answered by either "yes" or "no". For example, responding to a child's statement, "We yell all of the time so it's hard to think of like one single fight" with "You yell all of the time?".

b) Expansion paraphrasing occurred when the interviewer restated the child's utterance and continued to ask for more information with an open-ended prompt. For example, in response to a child's statement "I told them all the bad stuff they did to me" an interviewer may say, "Tell me more about the bad stuff they did to you".

c) Simple paraphrasing occurred when the interviewer simply restated what the child said without the use of intonation to make it a question and without adding an open-ended prompt. For example, if the child stated, "I made it up" the interviewer responded, "You made it up".

d) Summary paraphrasing occurred when the interviewer summarized several items which the child previously stated. For example, a child stated, "I went to the band concert at 7:00 because it started at 8:00 and then we performed for all the people. I got home at 10:00", and the interviewer responded "So you went to the concert at 7:00, started playing at 8:00, and got home by 10:00”.

\footnotetext{
${ }^{1}$ Coders used punctuation to determine how to code each interviewer utterance. Inter-rater reliability was performed on punctuation on $5 \%$ of the transcripts and a percent agreement of $100 \%$ was found.
} 
Each paraphrase was also coded to see whether the interviewer distorted children's words when paraphrasing. A paraphrase was coded as 'accurate' if the paraphrase did not alter the original meaning of the child's statement (i.e., the child saying, "I went to cadets at nine" and the interviewer replying with "You went to cadets at nine."). An inaccurate paraphrase on the other hand, altered or opposed a child's previous statement (e.g., a child saying, "Because of his tone" and the interviewer replied with "Because of his tongue?").

Inter-rater reliability was established at $80 \%$ with transcripts from a similar study prior to beginning coding. To ensure that the coding was consistent over time, $15 \%$ of the transcripts were randomly selected and a percent agreement inter-rater reliability for the type of paraphrasing was calculated. The inter-rater reliability between the two coders was $87 \%$.

\section{Children's Responses}

In addition to recording the type of paraphrasing used by the interviewer, the number of details reported by the child in response to each paraphrase was coded using a widely used coding system (Lamb, Sternberg, \& Esplin, 2000; Roberts et al., 1999; Sternberg et al., 1996). If an interviewer paraphrased a child's statement and then immediately asked a different question the statement was excluded because the child did not have the opportunity to respond to the paraphrase (i.e., if the child said "I was playing at my friend's house" and an interviewer responded "You were playing at your friend's house. Now, tell me about the time you were visiting your uncle"). A detail was defined as a subject, verb, object, or other meaningful detail regardless of the accuracy of information, provided that the children were recalling the event in question. For example, 
a child's statement of "he took off my shirt" would be coded as four details: he, took_off, my, shirt. Details were not coded when the child repeated a statement or spoke off-topic. Proportional scores were created to control for the number of prompts used in each interview. For example, the total number of details reported in response to expansion paraphrasing was divided by the total number of expansion paraphrases used in that interview.

Since the yes/no paraphrases created a question for the child to respond to, all responses to yes/no paraphrases were also further classified into one of five categories. The child either agreed, disagreed, expanded on their statement, did not respond, or said "I don't know" in response to the yes/no paraphrase. Proportional scores were computed to control for the number of yes/no paraphrases used in each interview (e.g., the total number of disagreed responses were divided by the total number of yes/no paraphrases in that interview).

Finally, in order to assess whether children corrected inaccurate paraphrases made by interviewers, the number of times children corrected inaccurate statements was counted.

Inter-rater reliability for the number of details reported was also established at $80 \%$ with transcripts from a similar study prior to beginning coding. A percent agreement inter-rater reliability was calculated for the details reported by children with a random selection of $15 \%$ of the transcripts to ensure the coding was consistent over time. Interrater reliability was calculated at $90 \%$ accuracy. These agreement figures are similar to those reported in other research of this type (e.g., Evans \& Roberts, in press; Roberts et al., 1999; Sternberg et al., 1996). 


\section{Results}

How often do investigative interviewers use each style of paraphrasing?

Preliminary analyses revealed that, due to the extremely low frequency of summary paraphrasing (used just 42 times across the 125 interviews, on average less than one time per interview; $M=.69, S D=1.51$ ), it was found to be significantly different from all other conditions. Thus, summary paraphrasing is excluded from future analyses.

To assess whether interviewers favored one style of paraphrasing over another, a 3 (paraphrasing condition: expansion, yes/no, simple) x 4 (child age in years: 4-6, 7-9, 10-12, 13-16) repeated measures ANOVA was performed on the number of paraphrases used. A significant main effect of paraphrasing condition was found, $F(2,222)=27.66, p$ $<.05, \eta 2=.20$. Follow-up LSD pairwise comparisons revealed that expansion paraphrasing $(M=14.00, S D=.86)$ was used significantly more often per interview than yes/no $(M=10.25, S D=1.15)$ and simple $(M=8.78, S D=.84)$ paraphrasing, $p<.05$. In addition, yes/no paraphrasing was used significantly more often in each interview than simple paraphrasing, $p<.05$. There were no other main effects or interactions. See Table 1 for a summary of means.

\section{Which types of paraphrasing elicit the most detailed reports?}

To assess whether the length of children's responses (i.e., number of elicited details) differed based on the style of paraphrasing used, a 3 (paraphrasing condition: expansion, yes/no, simple) x 4 (age: 4-6, 7-9, 10-12, 13-16) repeated measures ANOVA was performed on the proportional scores. A significant main effect of paraphrasing condition was found, $F(2,196)=18.77, p<.001, \eta 2=.16$. Follow-up LSD pairwise comparisons indicated that expansion paraphrasing elicited almost twice as many details 
per paraphrase $(M=11.70, S D=10.69)$ than simple $(M=6.30, S D=12.00)$ and yes/no $(M=4.56, S D=4.62)$ paraphrasing, $p<.05$. No other significant differences were found. Consistent with our hypotheses, expansion paraphrasing elicited the greatest amount of details per prompt from children in comparison to simple and yes/no paraphrasing. Children's responses to accurate and inaccurate paraphrases in investigative interviews

Next, a 3 (paraphrasing condition: expansion, yes/no, simple) x 2 (accuracy of paraphrase: accurate, inaccurate) x 4 (age: 4-6, 7-9, 10-12, 13-16) repeated measures ANOVA was conducted on the proportional scores. Note that the dependent variable here is the type of paraphrase by the interviewer (rather than children's statements). A main effect of accuracy indicated that interviewers paraphrased accurately $(M=.94, S D=.01)$ significantly more often than inaccurately $(M=.06, S D=.01), F(1,98)=3110.66, p<$ $.001, \eta 2=.97$. In addition, an interaction between accuracy and paraphrasing condition was found, $F(2,196)=13.84, p<.001, \eta 2=.12$. Follow-up paired sample t-tests revealed that a significantly higher proportion of yes/no paraphrases were inaccurate $(M$ $=.11, S D=.20)$ compared to expansion $(M=.03, S D=.07)$ and simple paraphrasing $(M$ $=.05, S D=.12), t(118)=-4.20, p<.05$, and $t(110)=-2.79, p>.05$, respectively $($ see Table 2 for ANOVA table).

Children's responses to interviewers' inaccurate paraphrases were assessed to see whether children corrected interviewers' inaccurate paraphrases. Corrected ratio scores were created by dividing the total number of times each child corrected an inaccurate paraphrase by the total number of inaccurate paraphrases in that interview. Paired sample t-tests were performed to explore if there was a significant difference between whether 
children corrected inaccurate paraphrases or left them uncorrected. Children were three times significantly more likely to not correct an inaccurate paraphrase $(M=.75, S D=$ $.30)$ than to correct the interviewer $(M=.25, S D=.30), t(69)=6.88, p<.001$.

Given the high rate of inaccurate yes/no paraphrases and the finding that children were not likely to correct such distortions (the present investigation; Roberts \& Lamb, 1999), children's responses to yes/no paraphrases were assessed to evaluate the types of responses children provided. A 5 (response: agree, disagree, expand, no response, don't know) x 4 (age: 4-6, 7-9, 10-12, 13-16) repeated measures ANOVA) on the proportional scores. A main effect of response was found, $F(4,496)=42.73, p<.001, \eta 2=.26$. Pairwise comparisons with Bonferroni correction for multiple comparisons revealed that children were significantly more likely to agree $(M=.42, S D=.26)$ or expand their statements $(M=.36, S D=.27)^{2}$ than disagree $(M=.09, S D=.12)$, give no response $(M=$ $.10, S D=.19)$, or say "I don't know" $(M=.01, S D=.05), p$ s $<.05$. In addition, children were significantly more likely to disagree or give no response than say "I don't know", $p$ s $<.05$ in response to yes/no paraphrases.

\section{Discussion}

Improving the length and quality of children's reports ultimately contributes to thorough investigations of child maltreatment victims. The present study investigated the use of paraphrasing as an investigative interview technique to elicit the most complete reports possible from alleged maltreated children. Specifically, we assessed whether paraphrasing was spontaneously used in 125 investigative interviews, and how children responded to different styles of paraphrasing in investigative interviews. Results

\footnotetext{
2 A random sample of 10 interviews was coded to assess the mean number of details reported when a child gave an expansion response to a yes/no paraphrase. On average 8.32 details were reported $(S D=10.19)$.
} 
indicated that indeed paraphrasing was a technique relied on by the investigative interviewers in this sample. Overall, paraphrasing was used on average approximately 31 times per interview. There was also a significant pattern of preferences in the use of different paraphrasing styles. Interviewers were significantly more likely to use expansion paraphrasing than any other style of paraphrasing (yes/no, simple, and summary), an encouraging result given that interviewers had been trained to use this style of paraphrasing.

The accuracy of the interviewers' paraphrases was also assessed and revealed that interviewers accurately paraphrased children's utterances significantly more often than inaccurately (94\% of paraphrases were accurate representations of children's reports). However, yes/no paraphrasing was performed inaccurately more often than expansion or simple paraphrasing. One possibility for these findings is that yes/no paraphrasing may be used when clarification is needed or when an interviewer is not sure they heard the child correctly. Either way, the practice of yes/no paraphrasing runs the risk that children's reports are distorted.

Children's responses also varied based on the style of paraphrasing used by interviewers. Consistent with experimental studies (Evans \& Roberts, in press), children reported significantly more details per prompt in response to expansion paraphrasing than all other styles of paraphrasing. Perhaps the expansion paraphrasing is a superior technique because it conveys interest, rather than disbelief, and makes an explicit request for information. Children may feel that the interviewer is interested in what they have to say and want to hear more and thus, are motivated to give more details in response to expansion paraphrases. In addition, expansion paraphrasing explicitly asks for additional 
information from children whereas yes/no paraphrasing and simple paraphrasing merely implicitly request more information. To ensure the difference between expansion paraphrasing and yes/no paraphrasing was not simply due to the different question types an ad hoc analysis was performed. Specifically, to assess whether expansion paraphrasing is different from open-ended prompts a paired-sample t-test was performed comparing the number of details reported in response to open-ended prompts (e.g., "Tell me more") with expansion paraphrasing in a sub-sample of the interviews (50\%) was performed. Open-ended prompts were found to elicit significantly more details $(M=$ $513.40, S D=511.18)$ compared to expansion paraphrases $(M=171.92, S D=207.34)$, $t(49)=6.89, p<.01$, suggesting that open-ended prompts and expansion paraphrasing are different from one another. It appears as though since expansion paraphrasing is following something the child just said about the prompt, fewer details are reported. Given the difference between expansion paraphrasing and open-ended prompts the openended nature of expansion paraphrasing alone do not account for the difference between expansion paraphrasing and yes/no paraphrasing.

Given that yes/no questions tend to elicit short, often one word, responses (e.g., yes or no) the number of details elicited by yes/no paraphrasing was still fairly high $(M=$ 4.56), although still significantly lower than other forms of paraphrasing. Children were not simply responding with one word answers. This may be due to the interview itself was structured as an open-ended interview. It may be that when a yes/no paraphrase is used within an open-ended interview it is less "damaging", disrupting the flow of rapport between child and interviewer less than when interviews are more directed. The global effect of the open-ended interview may override the specific effect of individual prompts. 
Future studies are required to further investigate the global dimension of investigative interviews.

Children's responses to yes/no paraphrasing were further investigated to assess whether children would be more likely to agree, disagree, expand on their response or say "I don't know". Consistent with our hypothesis, the most popular responses was to agree with the yes/no paraphrase. Children also expanded their responses, despite the closed nature of these paraphrases. One possible explanation for this finding is that, overall, interviewer's paraphrases were accurate thus children were likely to agree with interviewers. However, interviewers were significantly more likely to paraphrase inaccurately when using yes/no paraphrasing than any other style of paraphrasing. Due to the higher likelihood of inaccurate paraphrases and children's tendency to agree, yes/no paraphrasing appears to be a potentially risky interviewing technique.

To evaluate how costly inaccurate paraphrases really are, the likelihood of children correcting inaccurate paraphrases was assessed. Consistent with Roberts and Lamb's (1999) findings, children were three times more likely to not correct an interviewer's inaccurate paraphrase than to correct the paraphrase. However, it should be noted that the sample of interviewers in the current study were much more likely to accurately than inaccurately paraphrase children's statements (the number of accurate paraphrases was not reported in Roberts and Lamb's 1999 study). Replication of these results is important to determine the prevalence of riskier types of paraphrasing (i.e., yes/no paraphrasing) in other samples of interviews. The interviewers in the current study were undergoing extensive training and so may have been motivated to "do a good job" and reflect regularly on their interviewing practices. 
Another important finding of the current investigation is that the effects of paraphrasing did not differ by the age of the child witness. These findings are consistent with our hypotheses and with research conducted by Lamb and colleagues on the NICHD interview protocol. The NICHD interview protocol includes some of the paraphrasing manipulations evaluated in the present study (i.e., cued invitations and a form of expansion paraphrasing) and has also been found to improve both younger and older children's reports. Thus, it appears as though paraphrasing is a technique that can be consistently utilized with all ages of children.

One of the major limitations of this and most field investigations is our inability to evaluate the accuracy of children's reports. Although the present study is able to inform us about the amount of information reported in response to the different paraphrasing prompts, we do not know if these additional details are accurate. An experimental study conducted by Evans and Roberts (in press) found that children provided six times the number of accurate details in response to expansion paraphrasing than yes/no paraphrasing. It is also important to note that in response to expansion paraphrasing, children provided a small but significantly higher number of inaccurate details than yes/no paraphrasing. However, this is consistent with findings that indicate that a higher number of inaccurate details are sometimes reported when there is an overall increase in the number of details reported (e.g., Kohnken, Milne, Memon, \& Bull, 1999; Roberts et al., 2004).

The results of the present investigation have important implications for investigative interviewers of alleged child abuse cases. In general, expansion paraphrasing successfully elicited a more detailed report from alleged child abuse victims 
than yes/no, simple, and summary paraphrasing. The use of expansion paraphrasing is, therefore, recommended as a useful technique for interviewers. The results also provide two cautionary notes: yes/no paraphrasing is best avoided, and it is important to accurately paraphrase children's utterances (Roberts \& Lamb, 1999; Evans \& Roberts, in press). Importantly, expansion paraphrasing was associated with detailed responses from children across a wide age range, thus showing that children have the capacity to provide detailed reports of their experiences. 


\section{References}

Edwards, C. A., \& Forman, B. D. (1989). Effects of child interview method on accuracy and completeness of sexual abuse information recall. Social Behavior and Personality, 17, 237-247.

Evans, A. D., Roberts, K. P. (in press). The effects of different paraphrasing styles on the quality of reports from young child witnesses. Psychology, Crime and Law

Fisher, R.P., \& Geiselman, R.E. (1992). Memory-enhancing techniques for investigative interviewing: The Cognitive Interview. Springfield, IL: Thomas.

Koriat, M., \& Goldsmith, A (1996). Monitoring and control processes in the strategic regulation of memory accuracy. Psychological Review, 103, 490-517.

Goodman G.S., \& Reed, D.S. (1986). Age differences in eyewitness testimony. Law and Human Behavior, 10, 317-322.

Gordon, B. N., Ornstein, P. A., Nida, R. E., Follmer, A., Crenshaw, M. S., \& Albert, G. (1993). Does the use of dolls facilitate children's memory of visits to the doctor? Applied Cognitive Psychology, 7, 459- 474.

Hershkowitz, I. (2001). Children's responses to open-ended utterances in investigative interviews. Legal and Criminological Psychology, 6, 49-63.

Hunt, J.S., \& Borgida, E. (2001). Is that what I said? Witnesses' responses to interviewer modifications. Law and Human Behavior, 25, 583-603.

Köhnken, G., Milne, R., Memon, A., Bull, R. (1999). The cognitive interview: A metaanalysis. Psychology, Crime \& Law, 5, 3-27. 
Lamb, M. E., Sternberg, K. J., \& Esplin, P. W. (2000). Effects of age and delay on the amount of information provided by alleged sexual abuse victims in investigative interviews. Child Development, 71, 1586-1596.

Lamb, M. E., Sternberg, K. J., Orbach, Y., Esplin, P. W., Stewart, H., Mitchell, S. (2003). Age differences in young children's responses to open-ended invitations in the course of forensic interviews. Journal of Consulting and Clinical Psychology, 71, 926-934.

McCauley, M.R., \& Fisher, R.P. (1995a). Facilitating children's eyewitness recall with the revised cognitive interview. Journal of Applied Psychology, 80, 510-516.

McCauley, M.R., \& Fisher, R.P. (1995b). Enhancing children's eyewitness testimony with the Cognitive Interview. In G. Davies, S. Lloyd-Bostock, M. McMurran, \& C. Wilson (Eds.). Psychology, Law, and Criminal Justice: International Developments in Research and Practice (pp. 127-134). Walter de Gruyter: Oxford, UK.

Orbach, Y., \& Lamb, M. E. (2000). Enhancing children's narratives in investigative interviews. Child Abuse \& Neglect, 24, 699-708.

Orbach, Y., Hershkowitz, I., Lamb, M.E., Sternberg, K.J., Esplin, P.W., \& Horowitz, D. (2000). Assessing the value of structured protocols for forensic interviews of alleged child abuse victims. Child Abuse \& Neglect, 24, 733-752.

Roberts, K. P., Lamb, M. E., \& Sternberg, K. J. (1999). Effects of timing of postevent information on preschoolers' memory of an event. Applied Cognitive Psychology, $13,541-559$. 
Roberts, K.P., Lamb, M.E., \& Sternberg, K.J. (2004). The effects of rapport-building style on children's reports of a staged event. Applied Cognitive Psychology, 18, 189-202.

Roberts, K.P., \& Lamb, M.E. (1999). Children's responses when interviewers distort details during investigative interviews. Legal and Criminological Psychology, 4, 23-31.

Roebers, C. M., \& Fernandes, O. (2002). The effects of accuracy motivation on children's and adults event recall, suggestibility, and their answers to unanswerable questions. Journal of Cognition and Development, 4, 415-443.

Roebers, C. M., Moga, N., \& Schneider, W. (2001). The role of accuracy motivation on children's and adults event recall. Journal of Experimental Child Psychology, 78, 313-329.

Samra, J., \& Yuille, J. C. (1996). Anatomically-neutral dolls: Their effects on the memory and suggestibility of 4- to 6-year old eyewitnesses. Child Abuse \& Neglect, 20, 1261-1272.

Saywitz, K.J., \& Snyder, L. (1996). Narrative Elaboration: test of a new procedure for interviewing children. Journal of Consulting and Clinical Psychology, 64, 13471357.

Seidler, K. M., \& Howie, P. M. (1999). Motivational factors in children's reporting of events: The influence of age and expected reinforcement contingency. Journal of Applied Developmental Psychology, 20, 101-118. 
Shrimpton, S., Oates, K., \& Hayes, S. (1998). Children's memory of events: Effects of stress, age, time delay and location of interview. Applied Cognitive Psychology, $12,133-143$.

Sternberg, K. J., Lamb, M. E., Esplin, P. W., Orbach, Y., \& Hershkowitz, I. (2002). Using a structure interview protocol to improve the quality of investigative interviews. In L. M. Mitchell, J. A. Quas, \& G. S. Goodman (Eds.). Memory and Suggestibility in the Forensic Interview. Personality and Clinical Psychology Series (pp. 409-436). Lawrence Erlbaum Associates Publishers: Mahwah, NJ, US. Sternberg, K.J., Lamb, M.E., Hershkowitz, I., Esplin, P. W., Redlich, A., \& Sunshine, N. (1996). The relation between investigative utterance types and the informativeness of child witnesses. Journal of Applied Developmental Psychology, 17, 439-451.

Sternberg, K.J., Lamb, M.E., Orbach, Y., Esplin, P.W., \& Mitchell, K.J. (2001). Use of a structured investigative protocol enhances young children's responses to freerecall prompts in the course of forensic interviews. Journal of Applied Psychology, 86, 997-1005. 
Table 1. Mean and Standard Deviation (SD) for the frequency of each type of paraphrasing

\begin{tabular}{lcc}
\hline Type of Paraphrase & Mean & SD \\
\hline Summary & 0.69 & 1.50 \\
Simple & 6.78 & 8.72 \\
Expansion & 14.10 & 9.65 \\
Yes/No & 10.34 & 12.09 \\
Total & 31.92 & 25.37 \\
\hline
\end{tabular}


Table 2. Condition x Accuracy x Age Repeated Measures ANOVA on Proportional Scores

\begin{tabular}{lcccc}
\hline Variable(s) & df & Mean Square & $\mathrm{F}$ & $\mathrm{p}$ \\
\hline Condition & 2 & 0 & 0.49 & 0.61 \\
Condition x Age & 6 & 0 & 0.35 & 0.91 \\
Error (condition) & 196 & 0 & & \\
Accuracy & 1 & 114.85 & 3110.66 & 0.00 \\
Accuracy x Age & 3 & 0.14 & 3.80 & 0.01 \\
Error (accuracy) & 98 & 0.04 & & \\
Condition x Accuracy & 2 & 0.49 & 13.84 & 0.00 \\
Condition x Accuracy x Age & 6 & 0.08 & 2.29 & 0.04 \\
Error(condition x accuracy) & 196 & 0.04 & & \\
\hline
\end{tabular}

